\title{
AÇÕES E ESTRATÉGIAS PARA O FOMENTO DE UMA ECONOMIA CIRCULAR: BOAS PRÁTICAS APLICAVÉIS AO SETOR DA CONSTRUÇÃO CIVIL
}

Milena Mota Costa $1^{2}$; Orlando de Oliveira Freitas Junior $2^{2}$; Elaine Pinto Varela Alberte $3^{1}$; Alex Pires Carneiro $4^{2}$; Jadi Tosta Iglesias Ventin $5^{2}$

${ }^{1}$ Universidade Federal da Bahia; Salvador/Bahia; elaine.varela@ufba.br

${ }^{2}$ Universidade Federal da Bahia; Salvador/Bahia

Resumo: A Economia Circular pressupõe modelo econômico que fomenta a redução do desperdício e o lucro sustentável. Cada vez mais buscada em todos os contextos industriais, é fundamental para o setor da construção civil, tendo em vista os grandes impactos ambientais que esta indústria produz (consumo de materiais, energia e água, e geração de resíduos). Este trabalho busca identificar, em escala mundial, ações e estratégias para o fomento da Economia Circular neste setor em especial. A partir de pesquisa bibliográfica, realiza o levantamento sistematizado de boas práticas adotadas em setores industriais diversos, classificadas em todos os níveis e desenvolvidas por ambos agentes, setor público e iniciativa privada, e que foram identificadas como aplicáveis ao setor da construção civil.

Palavras-Chave: Boas práticas; Economia circular; Construção civil..

\section{ACTIONS AND STRATEGIES TO FOSTER A CIRCULAR ECONOMY: GOOD PRACTICES APPLICABLES TO THE CIVIL CONSTRUCTION SECTOR}

\begin{abstract}
The Circular Economy presupposes an economic model that promotes waste reduction and sustainable profit. Increasingly sought in all industrial contexts, it is fundamental for the construction sector, given the major environmental impacts that this industry produces (consumption of materials, energy and water, and waste generation). This paper seeks to identify, worldwide, actions and strategies for the promotion of Circular Economy in this particular sector. Based on bibliographic research, it conducts a systematic survey of good practices adopted in various industrial sectors, classified at all levels and developed by both public and private sector agents, and which were identified as applicable to the construction sector.
\end{abstract}

Keywords: Good habits; Circular economy; Construction 


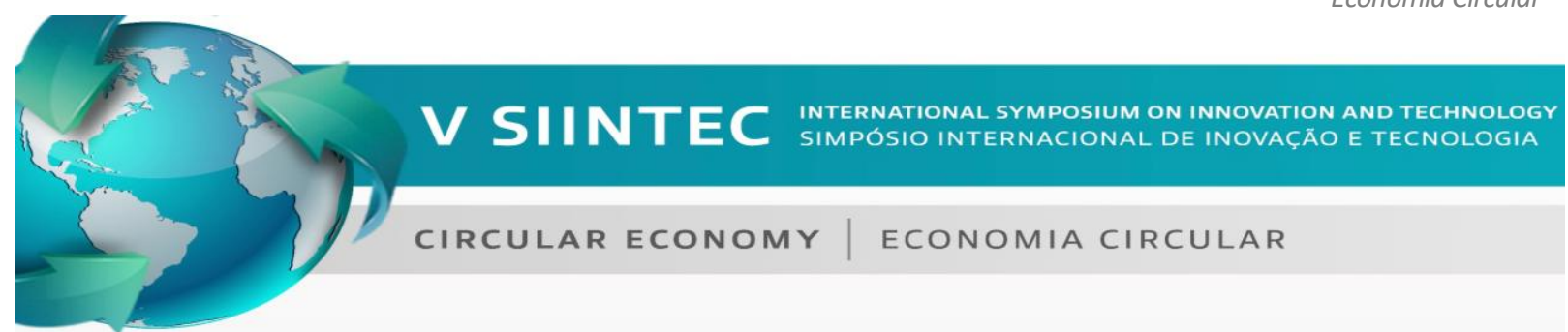

\section{INTRODUÇÃO}

No âmbito da Indústria da Construção Civil (ICC), a economia circular é uma abordagem para a minimização de resíduos ao longo de todo o ciclo da construção (i.e. [1]). Ela visa transformar a ICC através do desenvolvimento sustentável, onde premissas como reduzir, reutilizar, recuperar e reciclar materiais e energia, substituem o conceito de fim-de-vida, característico da economia linear.

O modelo econômico linear (extrair, transformar e descartar), ainda muito presente neste setor, já começa a apresentar significativos sinais de substituição, principalmente nos países desenvolvidos, graças à percepção de sustentabilidade e a consciência sócio-econômica de parte dos envolvidos. Portanto, economia circular é uma noção emergente que pode ser usada para transformar a economia linear em direção a uma abordagem circular maximizando os recursos (i.e. [1]).

Esse novo conceito promove a tomada de consciência no que se refere à redução da geração de resíduo na construção civil, destacando de forma engenhosa a atrativa economia financeira gerada ao longo de todo o processo, tanto na esfera pública quanto na iniciativa privada. Nesse contexto, as iniciativas podem proceder em duas vertentes, denominadas top-down e bottom-up. Segundo Rigamonti (i.e. [1]), o sentido top-down (de cima para baixo) envolve políticas e legislações especificas, infraestrutura de suporte e consciência social. Já o sentido bottom-up (de baixo para cima) utiliza modelos colaborativos de negócio, melhorias em design de produtos e cadeias de suplementos, além de tecnologia da informação.

O panorama brasileiro da ICC, em especial, vem gradativamente apresentando mudanças no que se refere à necessidade de melhoria de produtos, eficiência em reaproveitamento, responsabilidade e legalização dos devidos descartes e, sobretudo, renovação de ações. Ainda que incipiente e, em certos momentos, influenciadas por práticas ultrapassadas, o momento já inspira uma tendência sustentável e lucrativa, reportando-se aos princípios da Economia Circular (EC).

Deste modo, o presente artigo busca identificar ações e estratégias para o fomento do conceito de Economia Circular na ICC, a partir de levantamento sistematizado de boas práticas adotadas em setores industriais diversos. Apresenta como resultado análises sobre os mecanismos, benefícios, inovações e, principalmente, oportunidades de transformação que as boas práticas identificadas são capazes de gerar ao setor da construção.

\section{METODOLOGIA}

A presente pesquisa foi desenvolvida em duas etapas. A primeira etapa consistiu em uma pesquisa bibliográfica acerca do tema, reunindo conhecimentos no âmbito histórico e geral sobre EC e sua aplicação no setor da construção. Para esta revisão utilizaram-se artigos, periódicos de jornais, revistas, teses, dissertações, monografias, livros e relatórios de organizações. Como resultado, definiu-se os conceitos e as variáveis de análise adotadas para nortear a etapa seguinte, que objetivou o levantamento estruturado de boas práticas (BP) aplicáveis à ICC. Para a etapa de levantamento, foram identificadas 20 referências, que se destacaram com importantes contribuições sobre boas práticas que podem ser implementadas na construção civil. Deste conteúdo, foram identificadas boas práticas aplicadas 


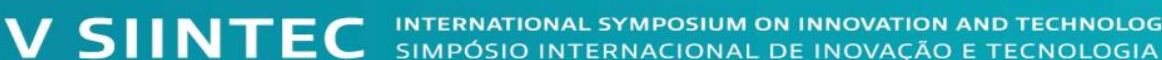

CIRCULAR ECONOMY

ECONOMIA CIRCULAR

/aplicáveis à ICC. Para a identificação das BPs adotou-se a definição da Plataforma de Boas Práticas para o Desenvolvimento Sustentável (i.e. [2]) que indica que uma boa prática consiste em uma técnica identificada e experimentada como eficiente e eficaz em seu contexto de implantação, para a realização de determinada tarefa, atividade ou procedimento ou, ainda, em uma perspectiva mais ampla, para a realização de um conjunto destes, visando o alcance de um objetivo comum.

As BPs, que podem ser adaptadas ou expandidas para esferas distintas, foram classificadas de acordo com os seguintes níveis de atuação:

Nível macro: De acordo com Ulgiati (i.e. [3]), retrata as economias nacionais, locais, regionais, como cidade, província, região, nação. Ou seja, ingerências políticas, podendo ser leis, programas de subsídio ou iniciativas que são implementadas pelo setor governamental;

Nível meso: Refere-se a um sistema integrado de agentes com interesses comuns, promovendo o desenvolvimento de parques eco-industriais, distritos e redes de simbioses industriais, bem como outras denominações de redes produtivas relacionadas (i.e. [4]). Ou seja, trata-se da atuação dentro de um conjunto sistemático que compartilha recursos ou produtos buscando o aumento da rentabilidade com redução significativa do impacto ambiental, cuja implementação pode contribuir diretamente para a sedimentação de uma economia sustentável (i.e. [4]).

$\bigcirc$ Nível micro: Segundo Ulgiati (i.e. [3]), ocorre em âmbito de empresa ou consumidor, sendo caracterizado como um processo único. Trata-se da ação realizada pela empresa para melhorar a circularidade de seu sistema de produção e também cooperar com outras empresas ao longo da cadeia de suprimentos para a obtenção de um padrão circular mais eficaz (i.e. [5]).

Dadas as definições, tem-se como resultado a análise estruturada das boas práticas fundamentadas nos princípios da economia circular aplicáveis ao setor da construção, utilizando-se as variáveis analíticas contidas nas Tabelas 2, 3 e 4.

\section{RESULTADOS E DISCUSSÕES}

A Tabela 1 apresenta o quantitativo de BPs identificadas na bibliografia selecionada, segundo nível de atuação e tipo de boa prática. Sendo esta ultima codificada do seguinte modo: BP1 - Leis e políticas públicas; BP2 - Criação de estrutura; BP3 - Programa de investimento, BP4 - Compartilhamento de recursos; BP5 - Associação de empresas; BP6 - Melhoria da durabilidade do produto; BP7 Métodos de produção enxutos/industrializados; BP8 - Estabelecimento de parcerias e convênios para a gestão de resíduos.

Do total de 191 BPs identificadas, o tipo mais evidenciado é a BP1, Leis e políticas públicas, correspondendo a cerca de $43 \%$. A BP6, melhoria da durabilidade do produto, é a que se apresenta em menor percentual, com cerca de $2 \%$. Quanto ao nível de atuação, as BPs no nível macro se destacam com aproximadamente 


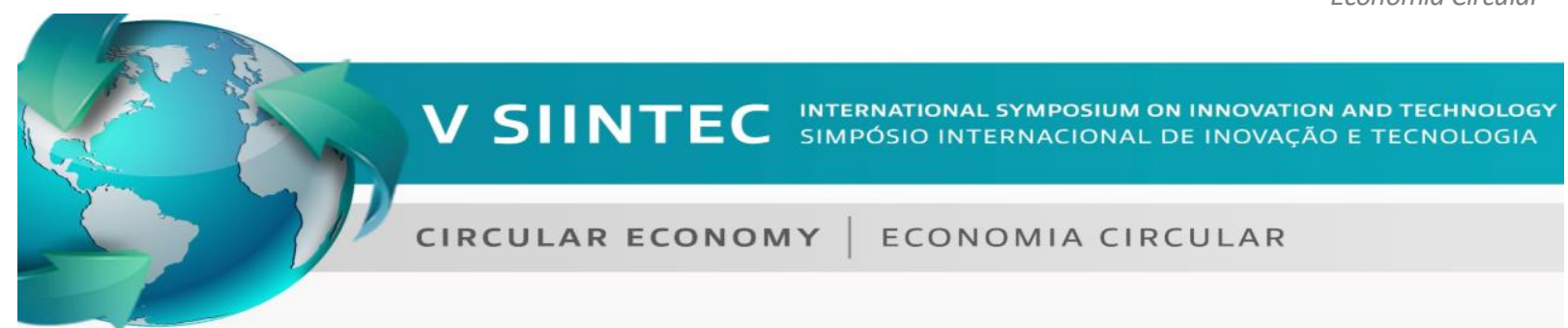

$52 \%$. Já nos níveis meso e micro têm-se, respectivamente, $22 \%$ e $26 \%$. As BPs foram destacadas conforme seu potencial de aplicabilidade dentro da ICC.

Diante disso, foram selecionadas 10 BPs relacionadas ao nível macro de atuação, sendo apenas 3 relacionadas diretamente ao âmbito da ICC. A Tabela 2 relaciona as BPs identificadas de acordo com as seguintes variáveis nominais: Âmbito de aplicação industrial, Mecanismo adotado e Local.

Tabela 1. Tipos de boas práticas identificadas em cada bibliografia

\begin{tabular}{|c|c|c|c|c|c|c|c|c|c|c|c|c|c|c|c|c|c|c|c|c|c|c|}
\hline \multirow{2}{*}{ 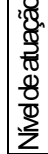 } & \multirow{2}{*}{$\begin{array}{c}\text { Tipode } \\
\text { Boa } \\
\text { Práica }\end{array}$} & \multicolumn{21}{|c|}{ Quantidade de Boas Práticas } \\
\hline & & Total & D1 & D2 & D3 & D4 & D5 & D6 & D7 & D8 & D9 & D10 & D11 & D12 & D13 & D14 & D15 & D16 & D17 & D18 & D19 & $D D O$ \\
\hline \multirow{3}{*}{$\begin{array}{l}\frac{0}{0} \\
\frac{\pi}{2}\end{array}$} & BP1 & 82 & 23 & 4 & - & 1 & 1 & 1 & 5 & 3 & - & - & 1 & 31 & 1 & - & - & 1 & 3 & 7 & - & - \\
\hline & BP2 & 11 & 6 & - & - & 1 & - & - & - & - & 2 & - & - & 2 & - & - & - & - & - & - & - & - \\
\hline & BP3 & 6 & - & - & - & 1 & - & - & - & - & - & - & - & 3 & 1 & - & - & - & 1 & - & - & - \\
\hline \multirow{2}{*}{$\begin{array}{l}\text { ᄋ } \\
\stackrel{\infty}{\Sigma}\end{array}$} & BP4 & 38 & 8 & 2 & - & - & - & - & - & - & - & 1 & - & - & - & 27 & - & - & - & - & - & - \\
\hline & BP5 & 4 & - & - & - & 2 & - & - & - & - & - & - & - & - & - & - & - & 1 & 1 & - & - & - \\
\hline \multirow{3}{*}{$\frac{\mathrm{d}}{\mathrm{U}}$} & BP6 & 3 & - & - & - & - & - & - & - & 1 & - & - & - & - & - & - & - & - & - & - & - & - \\
\hline & BP7 & 32 & - & - & 2 & - & - & - & - & - & 3 & - & - & - & - & - & 20 & - & - & - & 1 & 6 \\
\hline & BP8 & 15 & - & - & 1 & - & - & - & - & - & 1 & - & - & - & - & - & 6 & - & - & - & 3 & 3 \\
\hline
\end{tabular}

LEGENDA: D1 - Ulgiati (i.e. [3]), D2 -Rigamonti (i.e. [1]), D3 - Coca Cola (i.e. [7]), D4 - Hazell (i.e. [8]), D5 Macarthur (i.e. [9]), D6 - CONAMA (i.e. [10]), D7 - ABNT (i.e. [11-14]), D8 - Azevedo (i.e. [6]), D9 - Evangelista (i.e. [15]), D10 - Zhao (i.e. [16]), D11 - Adams (i.e. [17]), D12 - UNDP (i.e. [18]), D13 - EPA (i.e. [19]), D14 - Silva (i.e. [4]), D15 - Morató (i.e. [19]), D16 - Lozano (i.e. [20]), D17 - Nazaro (i.e. [21]), D18 - kruglianskas (i.e. [22]), D19 - Votorantin (i.e. [23]), D20 - MRV (i.e. [24]).

Tabela 2. Boas práticas identificadas em nível macro

\begin{tabular}{|c|c|c|c|c|c|}
\hline Boa prática & Código & Boa prática & Descrição & $\begin{array}{l}\text { Âmbito de } \\
\text { aplicação } \\
\text { industrial }\end{array}$ & Local \\
\hline \multirow{5}{*}{$\begin{array}{l}\text { BP1: Leis e } \\
\text { Políticas }\end{array}$} & BP1-MA-1 & $\begin{array}{l}\text { Lei da promoção da } \\
\text { economia circular }\end{array}$ & $\begin{array}{l}\text { Estabelece políticas para eliminação } \\
\text { de problemas de gestão da EC }\end{array}$ & Geral & China \\
\hline & BP1-MA-2 & $\begin{array}{l}\text { Lei sobre a promoção } \\
\text { dos recursos, economia } \\
\text { e reciclagem } \\
\end{array}$ & $\begin{array}{l}\text { Base para a reutilização de } \\
\text { materiais, para um sistema de taxas }\end{array}$ & Geral & Coréia \\
\hline & BP1-MA-3 & $\begin{array}{l}\text { Lei de prevenção da } \\
\text { poluição }\end{array}$ & $\begin{array}{l}\text { Redução da poluição por meio de } \\
\text { mudanças econômicas na produção, } \\
\text { operação e uso de materiais. }\end{array}$ & Geral & EUA \\
\hline & BP1-MA-4 & $\begin{array}{l}\text { Lei da minimização e } \\
\text { eliminação de resíduos }\end{array}$ & $\begin{array}{l}\text { Promove medidas para evitar a } \\
\text { geração de resíduos. }\end{array}$ & Geral & Alemanha \\
\hline & BP1-MA-5 & $\begin{array}{l}\text { Resolução CONAMA no } \\
\qquad 307^{5}\end{array}$ & $\begin{array}{l}\text { Norma jurídica que estabelece } \\
\text { diretrizes, critérios e procedimentos } \\
\text { para a gestão dos RCC. }\end{array}$ & $\begin{array}{l}\text { Construção } \\
\text { civil }\end{array}$ & Brasil \\
\hline \multirow{2}{*}{$\begin{array}{l}\text { BP2: Criação de } \\
\text { estrutura }\end{array}$} & BP2-MA-1 & $\begin{array}{l}\text { Construção de Eco- } \\
\text { cidades }^{1}\end{array}$ & $\begin{array}{l}\text { Programa governamental que } \\
\text { envolve centros urbanos e } \\
\text { industriais em projetos de simbiose, } \\
\text { com meta zero de emissão. }\end{array}$ & $\begin{array}{l}\text { Construção } \\
\text { civil }\end{array}$ & $\begin{array}{l}\text { Alemanha, } \\
\text { Suécia e } \\
\text { Reino } \\
\text { Unido }\end{array}$ \\
\hline & BP2-MA-2 & $\begin{array}{l}\text { Lei de implementação } \\
\text { de infraestrutura e } \\
\text { reciclagem }\end{array}$ & $\begin{array}{l}\text { Exige que os fabricantes operem } \\
\text { instalações de desmontagem, se } \\
\text { beneficiando diretamente da } \\
\text { recuperação de materiais e peças. }\end{array}$ & Geral & Japão \\
\hline $\begin{array}{l}\text { BP3: Programa } \\
\text { de investi-mento }\end{array}$ & BP3-MA-1 & $\begin{array}{l}\text { Incentivo financeiro para } \\
\text { viabilizar a comerciali- } \\
\text { zação de terras raras } \\
\end{array}$ & $\begin{array}{l}\text { Programa de subsídio para acelerar } \\
\text { a comercialização de tecnologias de } \\
\text { reciclagem }\end{array}$ & $\begin{array}{l}\text { Automobilís- } \\
\text { tica }\end{array}$ & Japão \\
\hline
\end{tabular}

${ }^{1}$ Ulgiati (i.e. [3]), ${ }^{2} \mathrm{Hazell}$ (i.e. [8]), ${ }^{3} \mathrm{Nazaro}$ (i.e. [21]), ${ }^{4} \mathrm{EPA}$ (i.e. [19]), ${ }^{5} \mathrm{CONAMA}$ (i.e. [10]). 
Observa-se que parte considerável das BPs selecionadas refere-se à criação de leis e políticas públicas que tratam da implementação da EC, as quais definem diretrizes para que as atividades desenvolvidas no território estejam condizentes com os princípios da sustentabilidade. Este cenário reforça a afirmação de Azevedo (i.e. [6]), que indica ser necessário investir em políticas públicas para estimular o rompimento com o modelo da economia linear.

De um modo geral, os Governos, buscando atuar de forma eficiente e inovadora, vêm estimulando essa mudança de atitude por meio de obrigatoriedades e/ou incentivos direcionados ao fortalecimento da EC. Ainda que muito possa ser feito no que diz respeito à criação de leis e políticas públicas, observa-se que as BPs na esfera pública não necessitam estar limitadas a isso. Destacam-se as BP2-MA-1 e BP2-MA-2, que se referem a programas governamentais com investimento público, que promovem a criação de estrutura física para o fomento da EC na região.

No nível meso de atuação, foram selecionadas 5 BPs, sendo todas relacionadas ao contexto de cooperação entre organismos, seja através do compartilhamento de recursos e estrutura, convênios ou associação de empresas. A Tabela 3 relaciona as boas práticas identificadas de acordo com as seguintes variáveis nominais: Mecanismo adotado, Setor Mercadológico e Local.

Tabela 3. Boas práticas identificadas em nível meso

\begin{tabular}{|c|c|c|c|c|c|}
\hline Boa prática & Código & Organismo & Descrição & $\begin{array}{c}\text { Setor } \\
\text { mercadológico }\end{array}$ & Local \\
\hline \multirow{4}{*}{$\begin{array}{l}\text { BP4: } \\
\text { Compartilhamento de } \\
\text { recursos }\end{array}$} & BP4-ME-1 & Synergy Park ${ }^{6}$ & $\begin{array}{l}\text { Empresas que compartilham } \\
\text { recursos, com co-geração. }\end{array}$ & $\begin{array}{l}\text { Alimentos } \mathrm{e} \\
\text { bebidas }\end{array}$ & $\begin{array}{l}\text { Aus- } \\
\text { trália }\end{array}$ \\
\hline & BP4-ME-2 & $\begin{array}{l}\text { Eco-Industrial de } \\
\text { Kalundborg }\end{array}$ & $\begin{array}{c}\text { Parceria entre nove empresas } \\
\text { públicas e privadas. }\end{array}$ & Refinaria & $\begin{array}{l}\text { Dina- } \\
\text { marca }\end{array}$ \\
\hline & BP4-ME-3 & $\begin{array}{l}\text { Parque Industrial } \\
\text { de Suzhou }\end{array}$ & $\begin{array}{c}\text { Cooperação bilateral entre } \\
\text { empresas para gestão de resíduos. }\end{array}$ & Geral & China \\
\hline & BP4-ME-4 & Porto Marghera ${ }^{6}$ & $\begin{array}{c}\text { Fomento a simbiose industrial e } \\
\text { local }\end{array}$ & $\begin{array}{l}\text { Indústria } \\
\text { química }\end{array}$ & Itália \\
\hline $\begin{array}{l}\text { BP5: Associação de } \\
\text { empresas }\end{array}$ & BP5-ME-1 & $\mathrm{ABRECON}^{7}$ & $\begin{array}{c}\text { Associação Brasileira de empresas } \\
\text { de reciclagem de RCC }\end{array}$ & Construção civil & Brasil \\
\hline
\end{tabular}

${ }^{6}$ Silva (i.e. [4]), ${ }^{7} A B R E C O N$ (i.e. [25]).

Ressaltam-se aqui iniciativas existentes em outros países que podem ser implementadas a nível nacional. China, Dinamarca, Austrália e Itália apresentam BPs relacionadas à criação de parques eco-industriais, prática em que ocorre o compartilhamento dos recursos e estruturas entre industrias (simbiose industrial) baseada nos fundamentos da EC (BP4-ME-1, BP4-ME-2, BP4-ME-3 e BP4-ME-4).

A China se destaca por liderar mundialmente o número de eco-parques industriais instalados (i.e. [3]). Já a Dinamarca, por ser o primeiro país a construir um eco-parque industrial (Kalundborg). Iniciado em 1959 e formado por nove empresas privadas e públicas, foi pioneiro na "simbiose industrial", em 1972, atraindo o interesse de todo o mundo (i.e. [4]).

Observa-se que as simbioses industriais identificadas ocorrem em diversos âmbitos da indústria. Apesar de não haver evidência, nesse estudo, de eco-parque industrial aplicado à ICC, observa-se que este conceito pode ser utilizado efetivamente na fabricação de produtos / matérias prima para a construção civil.

Em nível micro, foram identificadas 5 BPs, apresentadas na Tabela 4, segundo organismo, existência de recurso tecnológico, mecanismo adotado, 
proposta aplicada ao setor da construção civil, e etapa do ciclo de vida da construção relacionada ou passível de relação.

As BP7-MI-1 e BP8-MI-3 são aplicadas diretamente à ICC. A BP7-MI-1 referese à adoção de métodos construtivos que geram menos resíduos, nesse caso a parede de concreto, enquanto a BP8-MI-3 refere-se à implementação de programa de logística reversa com a política de retorno de embalagens vazias à fábrica.

As boas práticas BP6-MI-1, BP8-MI-1 e BP8-MI-2, por sua vez, são aplicadas a âmbitos industriais fora da ICC. Todavia, assim como nos níveis de atuação meso e macro, experiências bem sucedidas em outros contextos industriais podem servir de exemplo para medidas a serem desenvolvidas na ICC. A BP6-MI-1, por exemplo, pode ser interpretada ao setor construtivo, através da premissa de desenvolvimento /adoção de elementos construtivos (de caráter permanente ou temporário) com maior durabilidade. Premissa esta também adotada na BP7-MI-1, que compreende o uso de sistema de formas mais durável e menos gerador de resíduos, viabilizado em canteiro a partir da adoção de paredes de concreto como solução estrutural. As BP8-MI-1 e BP8-MI-2, por sua vez, são relacionadas ao estabelecimento de parcerias e convênios com empresas para gestão e destinação adequada de resíduos, podendo incluir ações de logística reversa com fornecedores.

Tabela 4. Boas práticas identificadas - nível micro

\begin{tabular}{|c|c|c|c|c|}
\hline Boa prática & Código & $\begin{array}{l}\text { Organis- } \\
\text { mo }\end{array}$ & Proposta aplicada a CC & $\begin{array}{l}\text { Ciclo de } \\
\text { vida }\end{array}$ \\
\hline $\begin{array}{l}\text { BP6: Melhoria da durabilidade } \\
\text { do produto }\end{array}$ & BP6-MI-1 & Philips $^{8}$ & $\begin{array}{c}\text { Desenvolvimento / adoção de elementos } \\
\text { construtivos mais duráveis }\end{array}$ & $\begin{array}{l}\text { Projeto e } \\
\text { design }\end{array}$ \\
\hline $\begin{array}{l}\text { BP7: Métodos de produção } \\
\text { enxutos/industrializados }\end{array}$ & BP7-MI-1 & $\mathrm{MRV}^{10}$ & $\begin{array}{l}\text { Uso de métodos construtivos mais enxutos / } \\
\text { industrializados }\end{array}$ & $\begin{array}{l}\text { Projeto e } \\
\text { design }\end{array}$ \\
\hline \multirow{3}{*}{$\begin{array}{l}\text { BP8: Estabelecimento de } \\
\text { parcerias e convênios para a } \\
\text { gestão de resíduos }\end{array}$} & BP8-MI-1 & $\begin{array}{l}\text { Procter \& } \\
\text { Gamble }^{8}\end{array}$ & $\begin{array}{c}\text { Estabelecer rede de parceiros para destinação } \\
\text { do RCC, incluindo logística reversa }\end{array}$ & $\begin{array}{l}\text { Construção } \\
\text { e demolição }\end{array}$ \\
\hline & BP8-MI-2 & $\begin{array}{l}\text { Coca- } \\
\text { Cola }\end{array}$ & $\begin{array}{l}\text { Desenvolvimento de embalagens } \\
\text { retornáveis/recicláveis (logística reversa) }\end{array}$ & $\begin{array}{l}\text { Construção } \\
\text { e demolição }\end{array}$ \\
\hline & BP8-MI-3 & $\underset{11}{\text { Votorantim }}$ & $\begin{array}{l}\text { Desenvolvimento de embalagens } \\
\text { retornáveis/recicláveis (logística reversa) }\end{array}$ & $\begin{array}{l}\text { Construção } \\
\text { e demolição }\end{array}$ \\
\hline
\end{tabular}

${ }^{8}$ Azevedo (i.e. [6]), ${ }^{9}$ Coca-Cola European Parners (i.e. 7]), ${ }^{10} \mathrm{MRV}$ (i.e. 24]), ${ }^{11}$ Votorantim (i.e. [23])

Experiências bem sucedidas demonstram que a transição para EC vem do envolvimento de todos os atores da sociedade e sua capacidade para ligar e criar padrões de colaboração e intercâmbio adequados. Histórias de sucesso também apontam a necessidade de um retorno econômico do investimento, a fim de proporcionar a motivação adequada para empresas e investidores (i.e. [3]). As iniciativas em nível micro podem propiciar economias significativas ao contexto empresarial, como a citada sobre a Procter \& Gamble (BP8-Ml-1), que gerou 1 bilhão em valor durante os últimos 5 anos, atingindo zero desperdício em $25 \%$ das suas instalações (i.e. [6]).

\section{CONCLUSÃO}

Em nível mundial é notável a existência de diversas boas práticas desenvolvidas a partir das premissas básicas da $\mathrm{EC}$, estando visivelmente distribuídas em todos os níveis (micro, meso ou macro). O ponto fundamental de tais ações bem sucedidas está no engajamento de todos os envolvidos, independente da esfera a qual pertença, seja ela do setor público ou de iniciativa privada. As boas práticas identificadas são, em parte considerável, de âmbitos industriais diferentes, o que não inibe a viabilidade de implementá-las na ICC. 
Cases de sucesso, novas tecnologias e produtos, maior consciência sobre os impactos ambientais e suas formas de prevenção, têm sido soluções amplamente apresentadas através das boas práticas em todos os níveis de envolvimento. Seja através da formulação de leis e política públicas, seja por meio das diversas iniciativas do setor privado, todas buscam um ponto de convergência para o reaproveitamento inteligente dos recursos. Isso, naturalmente, tem proporcionado um caminho mais promissor dentro dos processos particulares da ICC. Deste modo, o surgimento de novas redes e sistemas integrados de participação em todas as fases do ciclo construtivo, pode se tornar cada vez mais comum, proporcionando novo formato regenerativo que substituirá o modelo econômico linear.

Comprovando a efetiva economia de recursos em todas as fases, desde o planejamento, execução e retorno (fechamento do ciclo), evidenciando que mais do que uma possibilidade de redução de custos atrelada à preservação ambiental, a Economia Circular é uma proposta que vem se mostrando inteligente e lucrativa, se propondo a garanti melhores condições de vida para as gerações futuras.

\section{Agradecimentos}

Ao PIBIC/UFBA - UFBA, pela concessão de bolsas de pesquisa.

\section{REFERÊNCIAS}

${ }^{1}$ RIGAMONTI, Lucioa et al. Developing strategies for managing construction and demolition wastes in Malaysia based on the concept of circular economy. Japão, 2016.

${ }^{2}$ BOAS PRÁTICAS, Plataforma de Boas Práticas para o Desenvolvimento Susrentável. Conceitos e objetivos. Plataforma de Boas práticas para o desenvolvimento sustentável. Disponível em: < http://boaspraticas.org.br/-index.php/pt/saiba-mais/conceitos-e-objetivos>. Acesso em: 19 agos. 2019.

${ }^{3}$ ULGIATI, Sergio et al. A review on circular economy: the expected transition to a balanced interplay of environmental and economic systems. Beijing: JCP, 2016.

${ }^{4}$ SILVA, Willerson L. C. et al. Ecoparques industriais: uma revisão sistemática da literatura internacional. Brasil: ENEGEP 2016.

${ }^{5}$ WINKLER, H. Closed-loop production systems-A sustainable supply chain approach. Journal of Manufacturing Science and Technology, v. 4, n. 3, p. 243-246, 2011.

${ }^{6}$ AZEVEDO, Juliana Laboissière. A economia circular aplicada no Brasil: uma análise a partir dos instrumentos legais existentes para a logística reversa. Brasil: CONGRESSO NACIONAL DE EXCELÊNCIA EM GESTÃO, 2015.

${ }^{7}$ COCA COLA, Instituito. Isso não é um relatório de sustentabilidade. Coca Cola Brasil, Rio de Janeiro, jun. 2018. Disponível em: <https://www.cocacolabrasil.com.br/content/dam/journey/br/pt/private/pdfs/relatorio-de-sustentabilidade-coca-cola-brasil-2017.pdf>. Acesso em: 09 agos. 2019.

${ }^{8}$ HANZELL, Jonny et al. The circular economy in Japan. The Institution of Environmental Sciences, Abr. 2018. Disponível em: <https://www.the-ies.org/analysis/circular-economyjapan>. Acesso em: 09 agos. 2019.

${ }^{9}$ ELLEN MACARTHUR FOUDATION. Rumo à economia circular: o racional de negócio para acelerar a transição. Brasil: Ellen Macarthur Foudation, 2014.

${ }^{10}$ CONAMA, Conselho Nacional do Meio Ambiente. RESOLUÇÃo № 237, DE 19 DE dezembro DE 1997. Brasil, 1997. 
${ }^{11} A B N T$, NBR 15112. Resíduos da construção civil e resíduos volumosos - Área de transbordo e triagem - Diretrizes para projeto, implantação e operação. Brasil: Associação Brasileira de Normas técnicas, 2004.

${ }^{12}$ ABNT, NBR 15113. Resíduos sólidos da construção civil e resíduos inertess - Área de transbordo e triagem - Aterros - Diretrizes para projeto, implantação e operação. Brasil: Associação Brasileira de Normas técnicas, 2004.

${ }^{13}$ ABNT, NBR 15114. Resíduos da construção civil e resíduos volumosos - Área de transbordo e triagem - Diretrizes para projeto, implantação e operação. Brasil: Associação Brasileira de Normas técnicas, 2004.

${ }^{14} A B N T$, NBR 15115. Agregados reciclados de resíduos sólidos da construção civil Execução de camadas de pavimentação - Procedimentos. Brasil: Associação Brasileira de Normas técnicas, 2004.

${ }^{15}$ EVANGELISTA, Patricia $P$. A. et al. Alternativa sustentável para destinação de resíduos de construção classe A: sistemática para reciclagem em canteiros de obras. Brasil: Ambiente construído, 2010.

${ }^{16} \mathrm{ZHAO}$, Sidong et al. Chinese Industrial Park Planning Strategies Informed by American Edge Cities' Development Path - Case Study of China (Chongzuo)-Thailand Industrial Park. Procedia Engineering, p.832-840, 2017. Elsevier BV.

${ }^{17}$ ADAMS, Katherine et al. Circular economy in construction: current awareness, challenges and enablers. ICE Publishing 2017.

${ }^{18}$ UNITED NATIONS DEVELOPMENT PROGRAMME (UNDP). Human Development Indices and Indicators 2018 Statistical Update. New York, 2018.

${ }^{19}$ EPA. Resumo da Lei de Prevenção da Poluição. Agência de Proteção Ambiental dos Estados Unidos, Jan. 2017. Disponível em: < https://www.epa.gov/lawsregulations/summary-pollution-prevention-act >. Acesso em: 09 agos. 2019.

${ }^{19}$ MORATÓ, Jordi et al. Situación y evolución de la economía circular en España. Espanha: COTEC, 2017.

${ }^{20}$ LOZANO, Rodrigo et. al. Towards a more Circular Economy: Proposing a framework linking sustainable public procurement and sustainable business models. ELSEVIER: Resources, Conservation and Recycling, 2016.

${ }^{21}$ NAZARO, Mariane S. et al. Economia circular - uma abordagem inicial e sugestões para aplicação na gestão pública brasileira. Brasil: GCV, 2018.

${ }^{22}$ KRUGLIANSKAS, Isak et al. A Economia Circular no contexto europeu: Conceito e potenciais de contribuição na modernização das políticas de resíduos sólidos. Brasil, 2014.

${ }^{23}$ VOTORANTIM, Votorantim SA. Relatório Anual, São Paulo, 2018. Disponível em: <http://www.votorantim.com/relatorioanual/>. Acesso em: 15 agos. 2019.

${ }^{24}$ MRV, MRV Engenharia. Relatório de Sustentabilidade, Belo Horizonte, 2018. Disponível em: <https://www.mrv.com.br/sustentabilidade/upload/Publicacoes/ mrv2019030720190715161117.pdf>. Acesso em: 15 agos. 2019.

${ }^{25}$ ABRECON, Associação Brasileira para Reciclagem de Resíduos da Construção Civil e Demolição, São Paulo, 2019. Disponível em: < https://abrecon.org.br/>. Acesso em: 16 agos. 2019.

${ }^{26}$ ELLEN MACARYHUR FOUDATION. Towards the circular economy. Ellen Macarthur Foudation, 2010. 